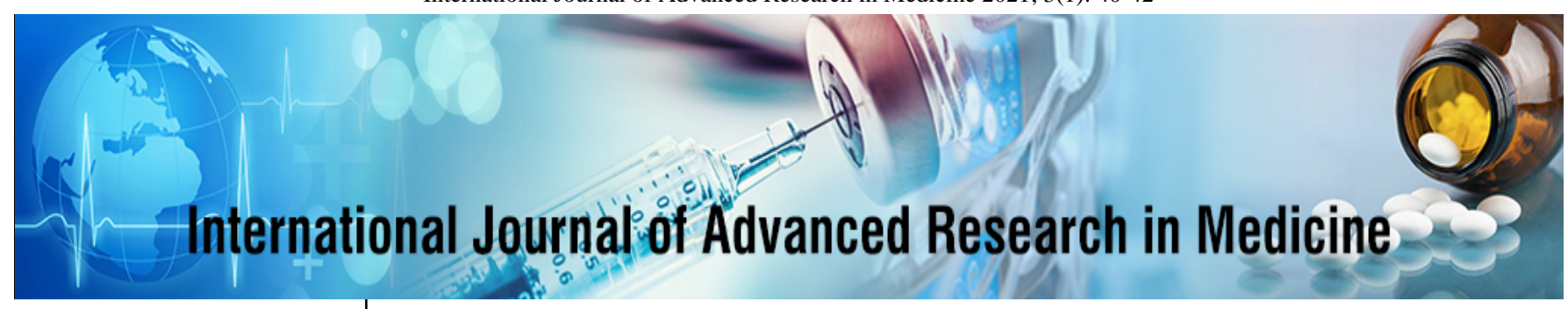

E-ISSN: 2706-9575

P-ISSN: 2706-9567

IJARM 2021; 3(1): 40-42

Received: 15-01-2021

Accepted: 01-02-2021

Dr. Balasaheb G Shelke Assistant Professor, SSPM Medical College \& Lifetime Hospital, Padve, Maharashtra, India
Corresponding Author: Dr. Balasaheb G Shelke Assistant Professor, SSPM Medical College \& Lifetime Hospital, Padve, Maharashtra, India

\section{HR-CT and mortality in COVID-19}

\author{
Dr. Balasaheb G Shelke
}

DOI: https://doi.org/10.22271/27069567.2021.v3.i1a.288

\section{Abstract}

The estimated mortality rate of COVID-19 is $3.5 \%$ on a global-wide scale, which is relatively low compared with the SARS-CoV and MERS-CoV. However, it varies by location, the intensity of transmission, and infection prevention and control measures. The mortality reached up to $6 \%$ in Wuhan (early epicenter area) in the early stage of the outbreak, and current $12.7 \%$ in Italy due to the rapid increase in the number of infections and shortage of medical resources. What is more, the mortality in critically ill patients has been reported as high as $60 \%$. In this context, early identification of risk factors for poor prognosis, accurate evaluation of disease severity and monitoring disease progression will be essential to reduce the mortality rate of patients with COVID-19. Chest CT imaging plays a valuable role in the screening and dynamic evaluation of patients with COVID-19. This study puts in an effort to find whether the CT scores can be associated with the outcomes of the disease.

Keywords: HR-CT, mortality, covid-19

\section{Introduction}

In October 2019, the first cases of new coronavirus disease (COVID-19) caused by severe acute respiratory syndrome coronavirus 2 (SARS-CoV-2) was initially reported in Wuhan City, China. The disease was then rapidly sweeping through the whole country and has spread to the other more than 150 countries and territories around the world. In March, 2020, the World Health Organization (WHO) declared the novel coronavirus outbreak to be a pandemic ${ }^{[1]}$ and as of now millions of cases are reported and more than half a million deaths have been recorded only in our country.

The infection of SARS-CoV-2 shares highly homological features with SARS-CoV, and causes acute, lethal respiratory pneumonia with typical clinical symptoms of fever and cough. According to an earlier report by WHO, most patients $(80 \%)$ experienced mild to moderate illness, and about $14 \%$ experienced severe disease and $5 \%$ were critically ill ${ }^{[2,3]}$. The estimated mortality rate of COVID-19 is $3.5 \%$ on a global-wide scale, which is relatively low compared with the SARS-CoV and MERS-CoV ${ }^{[4]}$. However, it varies by location, the intensity of transmission, and infection prevention and control measures. The mortality reached up to $6 \%$ in Wuhan (early epicenter area) in the early stage of the outbreak, and current $12.7 \%$ in Italy due to the rapid increase in the number of infections and shortage of medical resources. What is more, the mortality in critically ill patients has been reported as high as $60 \%{ }^{[5]}$. In this context, early identification of risk factors for poor prognosis, accurate evaluation of disease severity and monitoring disease progression will be essential to reduce the mortality rate of patients with COVID-19.

Chest CT imaging plays a valuable role in the screening and dynamic evaluation of patients with COVID-19 [6,7]. This study puts in an effort to find whether the CT scores can be associated with the outcomes of the disease.

\section{Aims and Objectives}

To find whether the CT scores can be associated with the outcomes of the disease.

\section{Materials and Methods}

This study was done in the Department of Pulmonology, SSPM Medical College \& Lifetime Hospital Padve. The study was done from April 2020 to March 2021. One hundred twenty one cases were chosen who had landed in the pulmonology Department. 


\section{Exclusion criteria}

Patients with other co-morbidities, so that there was no bias Patients who are immunosuppressant and chemotherapy.

The patients who underwent CT scanning, the radiographs were examined a CT score was given. Then the conditions of the patients were evaluated after a month and have been reported.

\section{Results}

Table 1: Age

\begin{tabular}{|c|c|c|}
\hline Total & Mean Age & SD \\
\hline 60 & 35.67 years & \pm 8.17 years \\
\hline
\end{tabular}

Table 2: Sex Distribution

\begin{tabular}{|c|c|c|}
\hline Total & Male & Female \\
\hline 121 & 78 & 43 \\
\hline
\end{tabular}

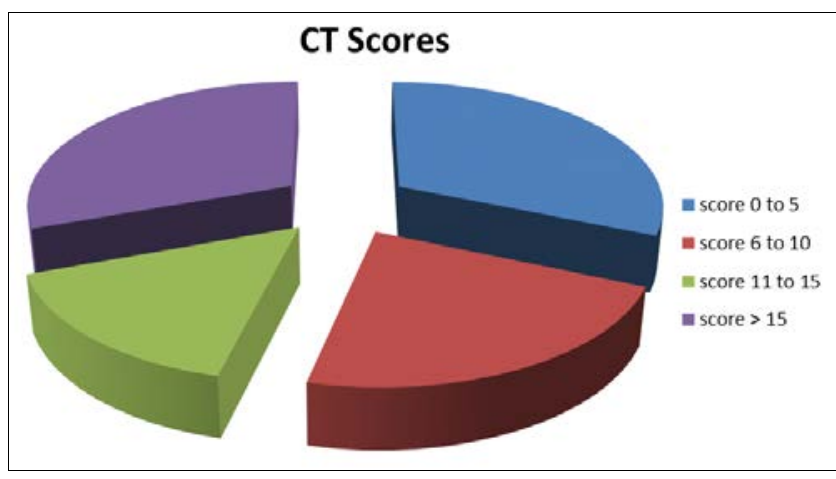

Graph 1: Initial CT scan

Table 3: After a month

\begin{tabular}{|c|c|}
\hline CT-Scores & Mortality \\
\hline $0-5$ & Nil \\
\hline $06-10$ & Nil \\
\hline $10-15$ & 1 \\
\hline$>15$ & 4 \\
\hline
\end{tabular}

Table 4: Association

\begin{tabular}{|c|c|c|c|}
\hline Total & CT-Scores & Mortality & p-value \\
\hline $37(31 \%)$ & $>15$ & 4 & $<0.001$ (Not sig) \\
\hline
\end{tabular}

Even though the association was not found but it is clear that more number of deaths was accounted in the group where the initial CT score was more. When the case fatality rate of the disease would be taken into consideration it would be very significant.

\section{Discussion}

Chest CT imaging plays an important role in the diagnosis and dynamic evaluation of COVID-19. Typical imaging features of multiple ground-glass opacities and/or consolidations in patients with COVID-19 pneumonia have been detailedly described in previous reports ${ }^{[8,9]}$. Even though the pathogenesis of SARS-CoV-2 infection is not fully understood, diffuse alveolar injury and progressive respiratory failure caused by SARS-CoV-2 is the leading cause of death in severe patients with COVID-19 ${ }^{[10]}$. From the current study, we found GGO and GGO with consolidation were the most predominant imaging features in patients who died from COVID-19, which is correlated with the pathological findings of COVID-19 that severe inflammatory exudation in intra-alveolar spaces and hyaline membrane formation ${ }^{[10,11]}$. The severity score of lung involvement in patients who died from COVID-19 was also significantly greater than that in patients with mild to moderate COVID-19 $(12.97 \pm 5.87 \text { vs. } 7 \pm 4)^{[6]}$. What is more, the mild-moderate correlation between chest CT severity scores and systemic inflammation activation was also preliminarily demonstrated in this study. Therefore, the imaging features and dynamic changes could provide the most direct evidence for assessing the severity of the disease and the prognosis. The outbreak of COVID-19 has had a strong impact worldwide. Almost all countries have suffered huge losses in health, society and economy. Our results may be potential risk factors to identify patients with poor prognosis, help clinicians to provide earlier interventions for these patients, and improve their survival rate.

There were some limitations in this present study. First, there was no control group included in this study. Thus, it is hard to evaluate the exact value of chest CT imaging in identifying the risk factors of poor prognosis as compared with the other clinical and/or laboratory parameters. A casecontrol study needs to be done shortly. Second, because many patients were transferred from the other hospitals, their early chest CT images were not available for us. In addition, some patients did not have follow-up chest CT scans due to critically ill conditions.

\section{Conclusion}

HR-Ct is can fairly predict the natural history but cannot be one hundred percent reliable. This when combined with the other biochemical markers, the prediction can be better.

\section{References}

1. World Health Organization (WHO).

2. Wu Z, McGoogan JM. Characteristics of and Important Lessons from the Coronavirus Disease 2019 (COVID19) Outbreak in China: Summary of a Report of 72314 Cases from the Chinese Center for Disease Control and Prevention. JAMA, 2020.

3. Situation Report. https://www.who.int/docs/defaultsource/coronaviruse/situation-reports/20200314-sitrep54-covid-19.pdf?sfvrsn=dcd46351_2.

4. Munster VJ, et al. A Novel Coronavirus Emerging in China-Key Questions for Impact Assessment. N Engl J Med. 2020;382(8):692-694. 10.1056/NEJMp2000929.

5. Yang $\mathrm{X}$, et al. Clinical course and outcomes of critically ill patients with SARS-CoV-2 pneumonia in Wuhan, China: a single-centered, retrospective, observational study. Lancet Respir Med, 2020.

6. Pan F, et al. Time Course of Lung Changes On Chest CT During Recovery From 2019 Novel Coronavirus (COVID-19) Pneumonia. Radiology. 2020, 200370.

7. Shi $\mathrm{H}$, et al. Radiological findings from 81 patients with COVID-19 pneumonia in Wuhan, China: a descriptive study. Lancet Infect Dis, 2020.

8. Xia W, et al. Clinical and CT features in pediatric patients with COVID-19 infection: Different points from adults. Pediatr Pulmonol, 2020.

9. Li Y, Xia L. Coronavirus Disease 2019 (COVID-19): Role of Chest CT in Diagnosis and Management. AJR Am J Roentgenol. 2020, 1-7. [Google Scholar]

10. $\mathrm{Xu} \mathrm{Z}$, et al. Pathological findings of COVID-19 associated with acute respiratory distress syndrome. Lancet Respir Med, 2020. 
11. Qian Liu, Rongshuai Wang, Guoqiang Qu, et al. Observation of corpse anatomy about coronavirus disease 2019 pneuminia decedent. Journal of Forensic Medicine. 2020;36(1):19-21.

12. Pan F, et al. Time Course of Lung Changes on Chest CT during Recovery From 2019 Novel Coronavirus (COVID-19) Pneumonia. Radiology. 2020, 200370.

13. Di Gennaro F, et al. Coronavirus Diseases (COVID-19) Current Status and Future Perspectives: A Narrative Review. Int J Environ Res Public Health. 2020, 17(8). 